Advances in technology and the neuroscience has made it possible for us to understand to a large extent the fundamentals behind the increasing behavioural problems in children. Focus will be on the aetiology - the root causes of changing behavioral profile of the young.

The study of the variations in the brain biology that allows cognitive abilities to develop through literature search. This needs to take centre stage in formulation of health care policies. Object should be to identify adverse factors.

Addressing the environmental factors that determine the biological processes involved in the development of criminal psychological traits, and corresponding adaptation of the clincial care pathways will be the most effective and cost efficient approach for the best outcome for the individuals and the society at large. Deterrants by way of force will only partially succeed in suppressing street crimes, as the call is to influence the brains of the developing children.

It is our professional responsisbility to aim for the best outcome for the individual child and the society at large.

\section{G625 COLLABORATION BETWEEN COMMUNITY CHILD HEALTH AND CHILD/ADOLESCENT MENTAL HEALTH SERVICES IN THE UK: RESULTS FROM A NATIONAL SURVEY}

1,2HF Ayyash, ${ }^{3} \mathrm{MO}$ Ogundele, ${ }^{4} \mathrm{~T}$ Schumm, ${ }^{5} \mathrm{O}$ Mitrofan. ${ }^{1}$ Dept of Paediatrics, Mid and South Essex University Hospitals Groups, Southend University Teaching Hospital, Southend, UK; ${ }^{2}$ Child and Adolescent Psychiatric Surveillance System, Royal College of Psychiatry, London, UK; ${ }^{3}$ Child Health Dept, County Durham and Darlington NHS FT, Darlington Memorial Hospital, Darlington, UK; ${ }^{4}$ Institute of Health and Wellbeing, University of Glasgow, Glasgow, UK; ${ }^{5}$ University of Exeter Medical School and Health Education South West (Peninsula), Exeter, UK

\subsection{6/archdischild-2020-rcpch.539}

Aims Neurodevelopmental and Emotional problems (NDEP), including behavioural and emotional problems, such as Attention Deficit Hyperactivity Disorder, Autism Spectrum Disorder, Tics disorder/Tourettes syndrome, Learning Disorders, developmental delay and development coordination disorder are commonly managed in the UK by the Community Child Health $(\mathrm{CCH})$ Paediatricians, often working within integrated specialist teams with allied health, social and educational professionals. Children and young people with NDED have three to four-fold increase in prevalence of co-occurring mental health disorders into adulthood. The current picture of integration between $\mathrm{CCH}$ and Child and Adolescent Mental Health $(\mathrm{CAMH})$ services in the UK is mixed and complex. We aimed to explore the experiences of $\mathrm{CCH}$ Paediatricians about working jointly with local CAMH services.

Method An online survey was conducted among CCH Paediatricians using a modified Child and Adolescent Psychiatrists Surveillance System (CAPSS) survey questionnaire, as part of a validation survey of CAPSS. The CAPSS focuses on surveillance of rare psychiatric conditions and $\mathrm{CCH}$ clinicians are considered to be able to play an important role in case ascertainment.

Results A total of 245 respondents working within $180 \mathrm{CCH}$ units across the UK completed the questionnaire, including Consultants (75\%), Specialist Associates (19.5\%) and Trainees (5\%). Only 27 respondents (11.6\%) reported working with young people aged $19-25$ years, often due to their complex disabilities, special education placement, delayed transition or lack of suitable adult services.
Participating Paediatricians' experiences suggest great variation in service provision and configuration for children of various ages and diagnosis across the UK. The commonest themes identified included increasing rate of $\mathrm{CCH}$ referrals from primary healthcare/other agencies as default, patients' experience of service fragmentation/bouncing between $\mathrm{CCH}$ and CAMHS. They also highlighted their challenges and desirability of joint-working with CAMHS.

Conclusion There are compelling historical and logical reasons to support a wider integration between $\mathrm{CCH}$ and CAMHS as optimal settings for providing effective and seamless high quality care to most children presenting with various $\mathrm{MH}$ and Neurodevelopmental disorders. Responses highlight the wide range of work $\mathrm{CCH}$ paediatricians are undertaking in the field of CYP mental health and therefore have essential roles in contributing to psychiatric surveillance.

\section{G626 USE OF LONG ACTING GUANFACINE IN ADHD AND ASSESSING THE COMORBIDITY AND PARENTS' PERCEPTIONS}

C Yemula, S Ajmal, R Sesham, T Banerjee. Community Paediatrics, Bedfordshire Community Health Services, Bedford, UK

\subsection{6/archdischild-2020-rcpch.540}

Aims A study was conducted to find out the clinical indications, prior stimulant use, associated comorbidity and parents/ carers' perceptions on the use of long acting guanfacine in children and adolescents with ADHD.

Methods The number of patients with ADHD on guanfacine over a period of 9 months were identified through IT support and their clinical records were accessed from our electronic system. Parents/carers' comments were also noted in relation to their child's progress.

Results 22 patients (17 boys and 5 girls) aged 7-16 years were commenced on guanfacine, with 50\% aged 13 years and above. All the patients had one or more associated comorbid conditions varying from sleep problems (50\%), autism spectrum disorder (40.9\%), anxiety (18\%), oppositional defiant behaviour (18\%), learning disability (9\%) and sensory processing problems (4.5\%). 21 patients previously received various formulations of either methylphenidate or amphetamine medications. However, one patient with a diagnosis of William's syndrome with cardiac problems was commenced on guanfacine as the 1 st option, following recommendation from a cardiologist. In 19 patients (86.3\%) the stimulant medication was not found to be effective, not tolerated in 2 patients (9\%) and not suitable in 1 patient $(4.5 \%)$. The stable/maintenance dose of guanfacine was noted to be $1 \mathrm{mg}$ for 5 patients (22.7\%), $2 \mathrm{mg}$ for 9 patients (40.9\%), $3 \mathrm{mg}$ for 6 patients $(27.2 \%)$ and $5 \mathrm{mg}$ for 2 patients (9\%). Towards clinical outcomes, 14 parents/carers (63.6\%) provided comments such as the child/adolescent is less aggressive, good symptom control, sleeping better, good start to school, best medication so far, much calmer and 'it is absolutely amazing!'

Conclusion Our study revealed that long acting guanfacine can be useful when the stimulant medication is not effective, not tolerated or not suitable. This complies with the current NICE guidelines. Despite a significant comorbidity, a high level of parents/carers' satisfaction is noted in our group. 